\title{
A Gestão do ConheCimento e a CAPACIDADE DE COMPETIÇÃO
}

\author{
André Eduardo Miranda dos Santos \\ MestreemAdministraçãodeEmpresas \\ FunionánioCLT VisaVale \\ andreedusantos@yahoocombr \\ Silvio Popadiuk \\ DatoremAdministracãodeEmpresas, Professordb \\ ProgramadePóśGraduacãoemAdministracãode \\ Empresasda UniveridadePresditeiamaMadkenzie \\ spopadik@madkeniebr
}

\section{RESUMO}

Com este artigo objetiva-se identificar se os benefícios das práticas de gestão do conhecimento são associados com capacidade de competição das empresas, segundo a perspectiva dos gestores. A abordagem teórica desenvolvida para atender a esse objetivo concentra-se nos conceitos derivados do modelo da ResaurcebasedV iew- RBV. Nesse modelo, a G estão do Conhecimento pode ser caracterizada como uma fonte de vantagem competitiva da firma. Foram pesquisadas oito empresas localizadas na cidade de São Paulo, de diferentes portes, de origem brasileira ou multinacional, e que afirmaram adotar práticas de gestão do conhecimento. 0 s dados foram coletados por meio de entrevistas não-estruturadas. O s resultados mostram evidências de que nas empresas estudadas os gestores reconhecem uma associação entre as práticas de gestão do conhecimento e a aquisição de uma vantagem competitiva.

Palavras-chave: G estão do Conhecimento. Capacidade de Competição. Vantagem Competitiva. ResarceBasedView

\section{ABSTRACT}

The djetive of this artide is to identify if the benfits of knowkedge managenent pradices that areassociated to the business' capadity of compeition, accordingtothemanages' vien Thetheertical approadh dadoped to reach this goal has foused on concepts deived from the resaurcebased vienmodd (RBV). IntheRBV modd, knontedge managenent can bedharacteized as a sarce of competitiveadvantage for thefirm Eight companies located in São Paulo with different sizes whoagreed toadqpt knowkedgemanagement pradices vere evaluated Data was colleted throughnon-structuredinterviens. The results showevidenes, besed on pereqtion of themanages, that these fimsidentify an assoiation between theknowkekgemamagement pratices and theacquistion of comptitiveadvantage

Key words: KnoweedgeManagement. Competition Capadity. CompetitiveAdvantage ResarceBasedView 


\section{INTRODUÇÃO}

A literatura sobre estratégias organizacionais sugere que a busca pelo diferencial competitivo está cada vez mais atreladaà atitude organizacional eà administração das atividades executadas pelas empresas, com a finalidade de atingir esse diferencial.

Nesse sentido, as disciplinas incorporadas ao estudo de estratégia continuam a se expandir, especialmente aquelas relacionadas ao "trabalhador do conhecimento" e à "sociedade da informação" (SPENCE R; GRANT, 1996). Inteligência, informação e conhecimento tornaram-se os núcleos dessas disciplinas. Uma nova terminologia foi incorporada à discussão sobre estratégia, evidenciando-se o conhecimento tácito, as competências organizacionais, os recursos intangíveis e a aprendizagem organizacional. E sses conceitos estão diretamente relacionados ao contexto interno das organizações e referem-se ao uso de seus recursos. Ao lado da teoria de custos de transação (WILLIAMSO N, 1991) e o modelo das cinco forças de Porter (1985), complementam uma equação que procura explicar os indicadores de competitividade das empresas.

De acordo com Wigg (2003), algumas medidas estão sendo tomadas em busca da efetividade e da valorização da gestão do conhecimento como uma ferramenta para atingir a excelência competitiva e o envolvimento das pessoas. No entendimento desse autor, o conhecimento é objeto de reflexão por filósofos ocidentais há muitos anos. Contudo, pelos mestres, artesãos e aprendizes, que dominam um sistema, o conhecimento é baseado em considerações pragmáticas e sistemáticas. Entretanto, lacunas permanecem ao se relacionar essas práticas com os resultados efetivos para as firmas, do ponto de vista da análise competitiva. Há uma constante indagação so bre o tipo de busca que as empresas demandam objetivando a aplicação dos conceitos de gestão do conhecimento. Elas podem estar mais preocupadas com a legitimação desse processo de gestão em vez de obter resultados efetivos decorrentes dessa forma de gerenciamento.

\section{Objetivos: geral e específicos}

É nesse sentido que o presente estudo foi desenvolvido. Seu objetivo foi o de identificar se ocorre uma associação entrea existência de práticas de gestão do conhecimento ea capacidade de competição das firmas. Em outras palavras, procurou-se responder se a existência de práticas de gestão de conhecimento constitui-se num fator que contribui para a capacidade de competição das firmas. D erivados do objetivo principal, seis objetivos específicos foram estabelecidos:

i) Identificar, na percepção dos informantes, como eles entendem 0 que seja o conceito de gestão do conhecimento;

ii) Identificar em que fase de aplicação esse processo se encontra na empresa;

iii) Identificar qual é objetivo estabelecido pela empresa para gerenciamento do conhecimento;

iv) Identificarqual áreaé responsável pelas iniciativas relacionadas à gestão do conhecimento

v) Identificar quais são os fatores que contribuem para a aplicação da gestão do conhecimento; vi) Identificar quais são os benefícios competitivos das práticas de gestão do conhecimento.

Para efeito de atender a esses objetivos, o presente estudo tem como suporte teórico três conceitos presentes na literatura de estratégia: i) o conceito de vantagem competitiva (BRITO; VASCO NCE LO S, 2004; GHEMAWAT, 1986; PORTER, 1980); ii) a ResarcebasedV iew(RBV) - visão baseada em recurSOS (BARNEY, 2001, 2002; DIERICKX e COOL, 1989, PETERAF, 1993), e iii) a teoria de gestão do conhecimento (TERRA, 2000; LIEBOVITZ, 1999; ALAVI; LEIDNER, 2001), e os fatores que contribuem para a sua aplicação.

\section{Contribuições}

D o ponto de vista das organizações, o estudo oferece a oportunidade para que as empresas atentem mais ao conhecimento que está sendo criado, disseminado, armazenado e utilizado, focando-0 numa estratégia voltada a resultados adquiridos frenteà concorrência e à competitividade. Pela perspectiva acadêmica, 0 estudo introduz novos elementos ligados à variável conheimentocomo ativo estratégico. Novas perspectivas surgem dos esforços para explicar as forças que direcionam a economia na "era do conhecimento", período caracterizado pela busca do conhecimento e pelas tentativas para incrementar os resultados no século XXI (WIIG, 2003).

\section{Estrutura do artigo}

$\mathrm{O}$ artigo é apresentado em outras quatro partes. Na primeira são apresentadas a discussão do conceito de vantagem competitiva e a conceituação de gestão do conhecimento. A segunda refere-se aos procedimentos metodológicos da pesquisa. Na terceira é apresentadaa análise e a interpretação dos resultados obtidos, subdivididos nos seis objetivos específicos. $\mathrm{Na}$ última parte são apresentadas as conclusões do estudo.

\section{FUNDAMENTAÇÃO TEÓRICA}

\section{Vantagem competitiva}

Há diferentes abordagens ligadas à ideia de vantagem competitiva. D e acordo com Brito e Vasconcelos (2004), sua base estáligada ao caráter do desempenho das empresas. Os autores afirmam que o conceito de vantagem competitiva não tem uma operacionalização detalhada e compartilhada pelos pesquisadores do tema. Contudo, a maioria dos autores desse campo reconhece que as empresas privadas se orientam pela procura da lucratividade crescente. Essas divergências no que tange à definição de vantagem competitiva são encontradas desde Ansoff (1965), que a definiu como a vantagem de perceber tendências de mercado à frente dos concorrentes. Essa conceituação ganhou maior popularidade a partir dos anos 80 , com a obra de Porter(1980), ao apresentar o seu modelo das cinco forças competitivas. Em 1986, G hemawat (1986) propõe que as fontes estruturais de vantagem competitiva poderiam ser encontradas em fatores ligados à inovação dos produtos, a processos de produção, ou às capacidades de markeingdas empresas. Porter (1985) argumenta que o objetivo e a medida de sucesso da estratégia passam a ser uma conquista de uma vantagem competitiva. Conceitua a chamada cadeia de valor como uma ferramenta para explicar a geração dessa vantagem. Afirma que a 
empresa conquistaria uma vantagem competitiva se executasse as atividades estrategicamente mais importantes da cadeia de valor de forma mais barata ou melhor do que a concorrência. G hemawat (1986) detalha que tais atividades podem decorrer de benefícios de tamanho, vantagens de acesso privilegiado a recursos e exercícios de opções que garantam flexibilidade estratégica.

Brito e Vasconcelos (2004) apresentam as duas vertentes com relação a essa conceituação: por um lado, os fenômenos de lucratividade persistentemente diferentes da média de mercado sendo naturais e desejáveis. Por outro lado, a homogeneidade natural e a perfeição dos mercados, em que tudo se estrutura para mostrar como os mercados convergem automaticamente para o equilíbrio, estabelecendo que fenômenos como as vantagens competitivas consistem em perturbações transitórias (RUMELT, 1991). A visão de Brito e Vasconcelos (2004) abrange uma definição constitutiva e operacional da vantagem competitiva, vendo-a como uma resultante de todos os fatores específicos e únicos da empresa em particular durante um período específico. Segundo os autores, a vantagem competitiva é concebida como uma influência líquida de todos os fatores particulares da empresa sobre o seu desempenho durante um período determinado, excluídas as influências de outros fatores, como a indústria e os fatores temporais.

Podem também ser feitas duas abordagens sobre as raízes dos estudos de vantagens competitivas. A primeira, segundo Bamey (2002), diz respeito à análise das ameaças e das oportunidades do ambiente, das forças e fraquezas da firma, e ao modelo das cinco forças competitivas de Porter, como as ferramentas, na abordagem da estrutura de desempenho em uma visão da organização industrial. Uma segunda visão, que, segundo Vasconcelos e Brito (2004), deu um tratamento teórico mais técnico ao conceito de vantagem competitiva, pode ser analisada sob a perspectiva da visão baseada em recursos (RBV ResarcebeselV ien).

Na explicação da diferença de desempenho entre firmas, Scherer e Ross (1991) descrevem a abordagem concentrada no setor industrial. Segundo os autores, vários aspectos ligados ao setor em que a firma atua contribuem para o seu desempenho. Entre esses aspectos, entendidos como associados à análise do ambiente externo, estão: i) os suprimentos de matériaprima; ii) a tecnologia; iii) os aspectos legais; iv) as condições de demanda relativas à elasticidade de preço; v) os métodos de compra e marketing vi) a estrutura de mercado, tais como 0 número de vendedores e de compradores; vii) as barreiras à entrada; ix) a estrutura de custos, ex) a forma como são conduzidos os processos inerentes ao comportamentos de preços e pesquisa, e desenvolvimento.

\section{Resource-based View}

Já a visão baseada em recursos (RBV) oferece uma estrutura própria para o estudo da vantagem competitiva. Enfatiza que os recursos específicos da empresa são determinantes para a geração de lucro econômico (WERNERFELT, 1984; BARNEY, 2001, 2002; DIERICKX; CO OL, 1989; PETERAF, 1993; BROUTHERS, BROUTHERS; WERNER, 2008).

Amit e Schoemaker (1993) afirmam que os recursos são todos aqueles fatores tidos ou controlados pela empresa, con- vertidos em produto e serviço final. Assim, segundo esses autores, a explicação do desempenho das firmas está relacionada aos recursos e às competências organizacionais. A competência consiste na capacidade da firma em transformar recursos, combinando processos organizacionais para atingir o final desejado. Eles ainda destacam as características desejáveis para que os recursos e as capacidades das firmas sejam fonte de vantagem competitiva: durabilidade, apropriabilidade, substitutos limitados, complementariedade, não imitação, raridade, baixa negociabilidade, e conectividade com os fatores estratégicos da indústria à qual a firma pertence. Nessa mesma linha, Barney $(2001,2002)$ também apresentaas características que um recurso deve apresentar para que possa ser uma fonte de resultados acima da média para a firma. Essas características, de alguma maneira, reforçam e abrangem aquelas apresentadas por Amite Schoemaker (1993). Assim, para Bamey $(2001,2002)$, um recurso pode ser fonte de vantagem competitiva se representa valor para o cliente, se éraro, se é difícil de imitar, e se pode ser explorado pela organização que o detém. E ssas quatro características são sintetizadas no acrônimo VRIO, derivado de value, ranity, non-imitabilityeorganizational capability.

Peteraf (1993) vincula as noções de rendas econômicas à noção de vantagem competitiva. Ela indica que avantagem competitiva deriva da heterogeneidade de recursos das empresas e da mobilidade imperfeita dos recursos entre elas, reforçados por limites ex antee ex postà competição (imitabilidade e substituição imperfeita de recursos).

É importante ressaltar que a RBV inclui as capacidades de gerenciamento do conhecimento da firma e demais ativos intangíveis como recursos que podem ser fontes de vantagem competitiva. Pela dificuldade de serem copiados e até mesmo identificados, são mais duradouros.

\section{Conceitos de conhecimento organizacional}

0 conhecimento se inicia em dados, definidos por D avenport e Prusak (1998) como conjuntos de fatos distintos e objetivos que, interpretados e dotados de relevância e propósito, resultam em informações (NONAKA; TAKE UCHI, 1997), as quais contribuem para o processo de geração do conhecimento individual ou organizacional. $O$ conhecimento torna-se, assim, um produto não puro e tampouco simples. É misturado com elementos formalmente estruturados e outros. Esses tendem a ser complexos e imprevisíveis. Envolvem experiências, valores, informações contextuais, e ingightsque possibilitam uma estrutura para a evolução e a incorporação de novas experiências e informação (DAVENPORT; PRUSAK, 1998; CHIA; HOLT, 2008).

Nesse conceito há diferentes dimensões para o conhecimento: uma visão mais formal e explícita, expressa em palavras e números, efacilmente comunicada e compartilhada sob a forma de dados brutos, fórmulas científicas, procedimentos codificados ou princípios universais. Trata-se do conhecimento explícito (NONAKA; TAKEUCHI, 1997). Por outro lado, o conhecimento concebido de forma pouco visível e exprimível, altamente pessoal e difícil de formalizar, transmitir e compartilhar com as demais pessoas é tido como conhecimento tácito (NONAKA;

CONTEXTUS Revista Contemporânea de Economia e Gestão. Vol.8 - № 1 - jan/ jun/ 2010. (21-32). 
KEUCHI, 1997; TSO UKAS, 2002; GOURLAY, 2004). O Quadro 1 sintetiza as características desses três conceitos.

\begin{tabular}{|l|l|l|}
\hline \multicolumn{1}{|c|}{ Dados } & \multicolumn{1}{|c|}{ Informações } & \multicolumn{1}{c|}{ Conhecimento } \\
\hline $\begin{array}{l}\text { Simples } \\
\text { observações sobre } \\
\text { o estado do } \\
\text { mundo }\end{array}$ & $\begin{array}{l}\text { Dados dotados de } \\
\text { relevância e } \\
\text { propósito }\end{array}$ & $\begin{array}{l}\text { Reflexão valiosa } \\
\text { da mente humana }\end{array}$ \\
\hline $\begin{array}{l}\text { Facilmente } \\
\text { estruturados }\end{array}$ & $\begin{array}{l}\text { Requerem unidade } \\
\text { de análise }\end{array}$ & $\begin{array}{l}\text { Inclui reflexão, } \\
\text { sintese e contexto }\end{array}$ \\
\hline $\begin{array}{l}\text { Facilmente } \\
\text { obtidos por } \\
\text { máquinas }\end{array}$ & $\begin{array}{l}\text { Exigem consenso } \\
\text { em relação ao } \\
\text { significado }\end{array}$ & $\begin{array}{l}\text { De difícil } \\
\text { estruturação e } \\
\text { captura por } \\
\text { máquinas }\end{array}$ \\
\hline $\begin{array}{l}\text { Frequentemente } \\
\text { quantificado }\end{array}$ & $\begin{array}{l}\text { Exigem } \\
\text { necessariamente a } \\
\text { mediação humana }\end{array}$ & $\begin{array}{l}\text { Frequentemente } \\
\text { tácito }\end{array}$ \\
\hline $\begin{array}{l}\text { Facilmente } \\
\text { transferível }\end{array}$ & $\begin{array}{l}\text { De difícil } \\
\text { transferência }\end{array}$ \\
\hline
\end{tabular}

Quadro 1: Caracteństicas de dados, informações e conhecimento

Fonte: Elaborado pelos autores.

Alavi e Leidner (2001) apresentam diferentes classificações de conhecimento dependendo do seu uso. Um exemplo é a afirmação de que o conhecimento pode ser classificado em declarativo (knowabaut) processual (knowhow', causal (knowivhy), condicional (knowiten) e relacional (knowwith), conforme pode serverificado no Quadro 2.

\begin{tabular}{|c|c|c|}
\hline \multicolumn{2}{|c|}{$\begin{array}{c}\text { Classificação do } \\
\text { conhecimento }\end{array}$} & Exemplos \\
\hline D eclarativo & Knowabaut & $\begin{array}{l}\text { Q ual matéria prima é mais } \\
\text { apropriada para determinado } \\
\text { produto }\end{array}$ \\
\hline Processual & Knowhow & $\begin{array}{l}\text { Como utilizar a matéria prima } \\
\text { para uma produção específica }\end{array}$ \\
\hline Causal & Knowfwhy & $\begin{array}{l}\text { Q uais tipos de efeitos impactam } \\
\text { a qualidade do produto ao se } \\
\text { alterar a especificação da matéria } \\
\text { prima }\end{array}$ \\
\hline Condicional & Knowrwhen & $\begin{array}{l}\text { Em que momento a } \\
\text { especificação da matéria prima } \\
\text { precisa ser conferida }\end{array}$ \\
\hline Relacional & Knowrwith & $\begin{array}{l}\text { Como determinado } \\
\text { componente humano ou } \\
\text { tecnológico interage com a } \\
\text { matéria prima }\end{array}$ \\
\hline
\end{tabular}

Q uadro 2: Classificação do conhecimento organizacional segundo seu uso

Fonte: Modificado a partir de Alavi e Leidner, 2001.

\section{Conceitos de gestão do conhecimento}

O termo "gestão de conhecimento" pode ser conceituado como a revisão dos principais processos, políticas, e ferramentas gerenciais e tecnológicas à luz de uma melhor compreensão do processo de geração/ criação, identificação, armazenamento, disseminação, compartilhamento e uso do conhecimento organizacional para gerar resultados econômicos para a empresa (TERRA, 2000). 0 autor completa que a gestão do conhecimento não é um projeto, mas a centralização de processos de gestão na variável conhecimento. D essa visão, sugere-se que a gestão do conhecimento é uma prática que se toma cíclica, que se inicia com a criação, passando pelas etapas enumeradas anteriormente, e chegando à ultima, que consiste no seu uso e/ ou aplicação que, em geral, associa-se com inovações incrementais ou radicais em produtos e/ ou serviços.

E isenhardte Santos (2002) definem a gestão do conhecimento como um processo sistemático, articulado e intencional, apoiado na geração, identificação, codificação, disseminação, apropriação, renovação e aplicação dos conhecimentos estratégicos na vida de uma organização que busca atingir a excelência organizacional. É a administração dos ativos de conhecimentos das organizações. E ssa gestão auxilia as organizações a tomarem as melhores decisões com relação às estratégias relativas aos clientes, concorrentes, canais de distribuição, ao ciclo de vida de produtos e aos serviços. É uma prática de agregar valor à informação e de distribuí-la dentro da organização.

Beckman (1999), na sua revisão de literatura, classifica que a gestão do conhecimento pode ser entendida sob qualquer uma das seis perspectivas: a conceitual, a gerencial, a tecnológica, a de implementação, a organizacional, e a processual.

Na perspectiva concitual destacam-se as definições, as dimensões e os princípios relacionados ao termo conhecimento. Na perspectiva gerenial, enfoca-se o papel da organização de proporcionar um ambiente favorável à criação do conhecimento, dando oportunidades de desenvolvimento a todos e criando mecanismos de mensuração, motivação, recompensa e premiação. A perspectiva teendógica consiste na visão de sistemas tecnológicos que dão suporte à gestão do conhecimento. 0 autor defende que a infraestrutura de sistemas de informação (SIs) deve ser levada em consideração, de forma a facilitar 0 compartilhamento do conhecimento. Salienta três características que os sistemas podem assumir: repositórios de conhecimento, sistemas de suporte de performance integrados e para transformação de conhecimento. A perspectiva de implemetaçãopontua os fatores preditivos de sucesso, assim como as barreiras e os desafios que poderão ser enfrentados, e as estratégias que podem ser adotadas na implementação da gestão do conhecimento. A perspectiva organizacional inclui a necessidade de se avaliar a estrutura organizacional, a orientação organizacional focada em gestão do conhecimento, os papéis e as responsabilidades dentro da organização voltada para o conhecimento, e a cultura corporativa (BECKMAN, 1999).

Earl (2001) propõe outra visão com base em revisões da literatura. Analisando as abordagens de diversos autores e combinando-as com a atuação de algumas empresas, 0 autor identificou que a gestão do conhecimento pode ser classificada sob o 
ponto de vista de sistemas, cartográfica, engenharia, comercial, organizacional, espacial e estratégica, cujas principais características são apresentadas no Q uadro 3.

\begin{tabular}{|c|l|}
\hline Categoria & \multicolumn{1}{|c|}{ D efinição / Foco } \\
\hline Sistemas & Captura de conhecimento \\
\hline Cartográfica & $\begin{array}{l}\text { Mapear, definir onde está localizado } \\
\text { cada conhecimento }\end{array}$ \\
\hline Engenharia & $\begin{array}{l}\text { O desempenho dos processos } \\
\text { melhora, com os conhecimentos } \\
\text { adequados. Seleção de } \\
\text { conhecimento. }\end{array}$ \\
\hline Comercial & $\begin{array}{l}\text { Comercialização do conhecimento; } \\
\text { transforma o conhecimento em } \\
\text { produto. }\end{array}$ \\
\hline Organizacional & $\begin{array}{l}\text { Uso de estruturas ou redes para } \\
\text { compartilhamento de } \\
\text { conhecimento. }\end{array}$ \\
\hline Espacial & $\begin{array}{l}\text { Criação de espaço para facilitar a } \\
\text { troca de conhecimentos. }\end{array}$ \\
\hline Estratégica & $\begin{array}{l}\text { Vê o conhecimento como uma } \\
\text { dimensão estratégica da organização. }\end{array}$ \\
\hline
\end{tabular}

Q uadro 3: Categonização de gestão do conhecimento, segundo Earl, (2001)

Fonte: Elaborado pelos autores, com base em Earl (2001).

Nas diferentes visões, a tecnologia da informação aparece sob algumas perspectivas. Sob o enfoque de objeto, Alavi e Leidner (2001) afirmam que o conhecimento pode ser armazenado e manipulado. Sendo assim, o papel da tecnologia da informação (TI) é unir, armazenar e transferir conhecimentos. Do ponto de vista da perspectiva processual, essas autoras afirmam que o papel da TI é prover ligações entre as fontes de conhecimento e criar um fluxo vasto e profundo do conhecimento. Quanto ao acesso de informação, a TI deve possibilitar mecanismos de pesquisa e, por fim, quanto às competências, o seu papel é suportar o desenvolvimento individual e organizacional.

A análise dessas várias definições permite concluir que a gestão do conhecimento pode ser redefinida como: Um processo de geração, aquisição, compartilhamento e distribuição de conhecimentos que envolve a combinação e o processamento de dados e informações, a transformação e o desenvolvimento de conhecimentos, a validação e a retenção desses conhecimentos, e a sua utilização.

\section{Gestão do conhecimento e a capacidade de competir}

Powell e D imaggio (1991) abordam que as empresas que adotam de maneira rápida o que eles chamam de inovações organizacionaissão comumente dirigidas pelo desejo de ampliar o seu desempenho. D entro desse enfoque, os autores caracterizam o isomorfismo como um processo de homogeneização das empresas pertencentes a determinado setor. Hawley (1968) relata que 0 isomorfismo é o constante processo que força uma unidade pertencente a uma população a querer se parecer com as demais unidades que enfrentam o mesmo conjunto de condições ambientais. Powell e Dimaggio (1991) ainda enfatizam que as organizações competem não apenas por recursos e clientes, mas também por poder político e legitimidade institucional. Trata-se até de um aspecto de ritual. Nesse caso, as empresas adotam suas inovações para atingir sua legitimidade e para demonstrar que estão, ao menos, tentando aprimorar as condições de trabalho.

A questão do modismo e da imitação (YANG; HYLAND, 2006) de capacidades e recursos leva ao que Barney (2002) denomina de paridade competitiva. As firmas, ao copiarem seus competidores, conseguem apenas se igualar a eles e não ultrapassá-los na corrida pelo desempenho. Segundo o autor, os recursos e as capacidades perdem o valor competitivo se outras firmas concorrentes entenderem e estiverem aptas a copiar ou adotar os mesmos recursos e práticas.

Nonaka e Takeuchi (1997), após anos de pesquisa sobre empresas japonesas, convenceram-se de que a criação do conhecimento é a principal fonte de sua competitividade internacional, principalmente pelo desenvolvimento de um conhecimento, definido anteriormente como conhecimento tácito. A única vantagem sustentável que uma empresa tem é aquilo que ela coletivamente sabe, aliado à eficiência com que ela usa esse conhecimento e à prontidão com que ela 0 adquire (DAVENPORT; PRUSAK, 1998).

Em uma visão mais abrangente, sugere-se que o conhecimento pode ser o recurso mais importante controlado por uma firma, e uma linha conhecida como a teoria baseada no conhecimento (KBV - Knoukedgebasedvien) tem se desenvolvido. Essa abordagem pode ser vista como uma extensão da teoria baseada em recursos ou como uma extensão das áreas de aprendizado organizacional e da teoria das organizações (EISENHARDT; SANTO S, 2002; ACED O, BARRO SO ; GALAN, 2006).

A revisão da literatura apresentada, envolvendo o conceito de vantagem competitiva, a ResarcebasedView - RBV, e a gestão do conhecimento, oferecem elementos para a fundamentação teórica necessária ao estudo aqui desenvolvido, cuja abordagem metodológica se apresenta na sequência.

\section{PROCEDIMENTOSMETODOLÓGICOS}

0 presente estudo é de caráter exploratório com umaabordagem qualitativa. Foram considerados dois construtos na pesquisa. 0 primeiro éa gestão do conhecimento, caracterizado por um conjunto de variáveis tais como: gestão de processos, rodízio de funcionários, universidade corporativa, técnicas de benhmarkinge disseminação de melhores práticas, ferramentas de tecnologia da informação, programas de inovação emelhoria contínua e práticas de pesquisa com o cliente. E sses elementos foram identificados como fatores que contribuem no ciclo da gestão do conhecimento envolvendo as etapas de geração, identificação, armazenamento, disseminação, compartilhamento e uso do conhecimento organizacional (TERRA, 2000). 0 segundo construto é a vantagem competitiva, que foi operacionalizada como a percepção dos gestores do impacto no desempenho da empresa em determinado período de tempo, excluindo as influências de outros fatores como a indústria e os fatores temporais (BRITO; VASCONCELOS, 2004).

A amostra foi composta de oito empresas selecionadas por conveniência devido à facilidade de acesso dos pesquisadores. As empresas foram selecionadas independentemente do 
setor, do porte, da origem geográfica, e de estarem situadas na cidade de São Paulo. O s sujeitos da pesquisa foram funcionários que lideravam projetos ou áreas relacionadas às práticas de gestão do conhecimento: gerentes, analistas e coordenadores de recursos humanos, diretores e gerentes de tecnologia da informação, engenheiros seniores, diretor de operações de manufatura. $\mathrm{O}$ Q uadro 4 resume tais características: bilidade. Nesse sentido, buscou-se identificar o real entendimento do assunto por parte do entrevistado, verificando se havia algum tipo de divergência entre o conhecimento do entrevistado e a conceituação teórica, com relação aos temas componentes do roteiro desenvolvido. Para os cinco demais tópicos, a pergunta era um tanto mais direta e não exigiu detalhamentos para a sua formulação no ato da entrevista.

\begin{tabular}{|c|c|c|c|c|c|}
\hline $\begin{array}{c}\text { Empresa } \\
\text { pesquisada }\end{array}$ & Porte & Atividade & Setor & Função do informante & Origem geográfica \\
\hline A & Grande & Engenharia civil & Indústria & Engenheiro sênior & Nacional \\
\hline B & Grande & Consultoria & Serviços & Gerente de conhecimento & Multinacional \\
\hline C & Grande & Comunicação & Indústria & Diretor de TI & Nacional \\
\hline D & Média & Metalúrgica & Indústria & Diretor de operações & Multinacional \\
\hline E & Média & Vale alimentação & Serviços & Coordenador de treinamento & Multinacional \\
\hline F & Pequena & Ventilação & Indústria & Gerente de marketing & Nacional \\
\hline G & Média & Autopeças & Indústria & Coordenador de treinamento & Multinacional \\
\hline H & Grande & Engenharia elétrica & Indústria & Gerente de informação & Multinacional \\
\hline
\end{tabular}

Q uadro 4: Caracterização das empresas pesquisadas

Fonte: Elaborado pelos autores, com base nos dados da pesquisa.

A coleta de dados foi realizada por entrevistas pessoais, utilizando-se de um roteiro semiestruturado com onze questões abertas, que permeavam os aspectos que direcionavam ao reconhecimento de atingimento de vantagens competitivas, elaborados a partir da revisão bibliográfica e da seleção preliminar que identificavam se o gestor reconhecia ou não a aplicação de práticas de gestão do conhecimento. Essa coleta de dados foi realizada em duas etapas. Na primeira, utilizou-se um roteiro preliminar e foi realizada por email ou telefone, buscando-se identificar as empresas cujos gestores reconheciam o uso de uma gestão baseada em conhecimento. Posteriormente, uma vez identificadas as empresas (seis no setor industrial e duas do setor de serviços), realizaram-se as pesquisas presenciais, utilizando-se um segundo roteiro semiestruturado mais detalhado. Esse segundo roteiro foi dividido em seis tópicos, segundo o que foi estabelecido como objetivos específicos, enumerados a seguir, e que consistiam na identificação: i) do que se entende por gestão do conhecimento; ii) da fase de gestão do conhecimento em que a empresa se encontrava; iii) do objetivo estabelecido pela empresa para gerenciamento do conhecimento; iv) da área responsável pelas iniciativas relacionadas à gestão do conhecimento; $v$ ) dos fatores que contribuem para a aplicação da gestão do conhecimento; vi) dos benefícios competitivos das práticas de gestão do conhecimento.

0 entendimento do que consistia o conceito de gestão do conhecimento, objeto do primeiro tópico do roteiro, focavase em itens relacionados às melhores práticas, ao monitoramento do ambiente, à universidade corporativa, ao Customer Reahhionship Managenent (CRM), à substituição dos colaboradores das funções, e às atividades da empresa que garantissem o comprometimento pessoal e o senso de responsa-
Todas as entrevistas foram previamente agendadas e gravadas com a autorização dos entrevistados. As gravações foram integralmente transcritas, tomando-se o cuidado de preservar as características originais da entrevista, atendendo ao disposto por Bardin (1997), que recomenda uma transcrição exaustiva e que conserve o máximo de informações. D urante a entrevista não foi citado o conceito teórico sobre o tema, evitando-se, assim, possíveis influências nas respostas dos entrevistados.

$\mathrm{Na}$ coleta dos dados secundários foram identificados os fatores citados anteriormente, que contribuem na aplicação da gestão do conhecimento: i) gestão de processos; ii) gestão de pessoas (o papel da liderança, o plano de carreira, os incentivos, a universidade corporativa e a motivação); iii) técnicas de bentmarking iv) tecnologia dainformação; v) inovação e melhoria contínua; vi) comunicação interna estruturada, e vii) pesquisa com o cliente. Na entrevista buscou-se identificar o que a empresa aplicava com relação a cada fator.

Porfim, buscou-se identificar se havia alguma prática, algum sistema ou procedimento que mensurasse o sucesso da iniciativa. Independentemente da resposta, questionou-se sobre a compreensão do gestor nos resultados obtidos com a aplicação de gestão do conhecimento e 0 atingimento ou não de uma fonte de vantagem competitiva.

A análise dos dados obtidos na etapa de coleta considera como unidade de registro o "tema", uma vez que estava se buscando nas informações dos gestores considerações que permeassem o estudo sobre gestão do conhecimento.

Considerando-se o processo amostral do estudo, não está garantida a validade externa dos dados obtidos. D essa forma, os resultados são válidos apenas para a amostra em questão. (RICHARD SO N et al., 1999). 


\section{ANÁLISE E INTERPRETAÇÃO DOS RESULTADOS}

O s dados coletados foram analisados pelo uso da técnica de análise de conteúdo proposta por Bardin (1997). Para a autora, a codificação corresponde a uma transferência do material, efetuada segundo regras precisas, dos dados brutos do texto. Tal transferência deve serfeita por recorte, agregação e enumeração, permitindo atingir uma representação do conteúdo, ou da sua expressão, suscetível de esclarecer o analista acerca das características do texto, que podem servir de índices.

\subsection{Conceito de gestão do conhecimento, fase de aplicação e objetivos}

O Q uadro 5 consolida a análise relativa aos três primeiros objetivos específicos estabelecidos neste trabalho. 0 primeiro envolveu o entendimento do gestor que conduzia as iniciativas de gestão do conhecimento com relação ao tema e o nível de aplicabilidade na companhia. Nesse sentido foram abordados três tópicos para direcionar a resposta dos entrevistados: melhores práticas, monitoramento do ambiente e substituição dos colaboradores, e uma perda do conhecimento da firma. A análise das respostas dos entrevistados revelou uma grande variação na definição. Ficou claro que ela dependia da área de atuação, do porte, da visão e da evolução da aplicação na empresa. D ois dos oito gestores entrevistados se mostraram equivocados com os conceitos abordados pela literatura. Eles os entendiam como gestão da informação e/ ou gestão da qualidade. Todos os entrevistados mostraram interesse na aplicação da iniciativa, embora cada um estivesse em um estágio diferenciado de uma implementação formalizada da gestão do conhecimento. Alguns estavam em fase de estudo e análise. 0 utros, ainda estavam em fase de compreensão dos conceitos, de implantação efetiva, de medição dos resultados ou de aperfeiçoamento dos fatores de contribuição. Nas empresas E e F não existiam iniciativas formais de gestão do conhecimento, apesar de ser identificados indícios pelos próprios gestores. Contudo, a empresa $\mathrm{E}$ estava em processo de análise e estudos. A empresa B se encontrava na fase de manutenção, e as demais $(A, C, D, G, H)$ se encontravam em fase de aprimoramento. Vale ressaltar que um dos entrevistados abordou a gestão do conhecimento como uma prática vinculada a três pilares: motivação, processos e tecnologia. A iniciativa nessa empresa buscava mapear as competências para saber o que cada um fazia dentro da organização, podendo se preparar para o futuro, focando nos impactos e enxergando onde éimportante aprimorar o conhecimento.

Embora tenham sido encontrados conceitos diferentes, pela análise de conteúdo, foram identificadas algumas similaridades nas visões dos entrevistados, conforme pode ser comprovado no Q uadro 5. Nesse mesmo Quadro identificam a respostas associadas aos objetivos de um processo de implementação da gestão do conhecimento na empresa. As respostas foram variadas. Envolviam a identificação de conhecimentos existentes na empresa, 0 apoio a processos de tomada de decisões, 0 aprimoramento de produtos, processos e serviços e/ ou simplesmente motivar os funcionários.

\begin{tabular}{|c|c|c|c|c|c|c|c|c|c|}
\hline $\begin{array}{c}\text { Conceitos sobre a } \\
\text { gestão do } \\
\text { conhecimento }\end{array}$ & A & B & C & D & $\mathbf{E}$ & $\mathbf{F}$ & G & $\mathbf{H}$ & Tota \\
\hline $\begin{array}{l}\text { Composição de } \\
\text { várias disciplinas }\end{array}$ & Sim & & & $\mathrm{Sim}$ & & & & Sim & 3 \\
\hline $\begin{array}{l}\text { Distribuição de } \\
\text { informação }\end{array}$ & Sim & & Sim & & & Sim & & & 3 \\
\hline $\begin{array}{l}\text { Compartilhamento } \\
\text { do conhecimento }\end{array}$ & $\operatorname{Sim}$ & & & & Sim & & & $\mathrm{Sim}$ & 3 \\
\hline $\begin{array}{l}\text { Administração do } \\
\text { conhecimento } \\
\text { adquirido por } \\
\text { colaboradores }\end{array}$ & & & & & & & Sim & Sim & 2 \\
\hline $\begin{array}{l}\text { Cultura } \\
\text { corporativa }\end{array}$ & & Sim & & & & & & & 1 \\
\hline
\end{tabular}

Objetivo da gestão do conhecimento

\begin{tabular}{|l|c|c|c|c|c|c|c|c|c|}
\hline $\begin{array}{l}\text { Identificar quem } \\
\text { possui o } \\
\text { conhecimento }\end{array}$ & Sim & & & & & Sim & Sim & $\mathbf{3}$ \\
\hline $\begin{array}{l}\text { Apoio à tomada de } \\
\text { decisão }\end{array}$ & & Sim & Sim & & & & Sim & $\mathbf{3}$ \\
\hline $\begin{array}{l}\text { Melhorar produto, } \\
\text { serviço ou } \\
\text { processo }\end{array}$ & Sim & Sim & & & & $\mathbf{2}$ \\
\hline $\begin{array}{l}\text { Motivação. Tornar } \\
\text { melhor lugar para } \\
\text { se trabalhar }\end{array}$ & & & & Sim & & & Sim & $\mathbf{2}$ \\
\hline $\begin{array}{l}\text { Atender às } \\
\text { regulamentações }\end{array}$ & & & & & Sim & & & $\mathbf{1}$ \\
\hline
\end{tabular}

Q uadro 5: Conceitos sobrea G estão do conhecimento e qual é seu objetivo

Fonte: Elaborado pelos autores, com base nos dados da pesquisa.

\section{2Áreas responsáveis edepartamentos- alvo das iniciativas}

0 quarto objetivo específico relacionava-se à identificação da área responsável pelainiciativa e o responsável imediato dela, assim como os departamentos alvo e a explicação por focar neste departamento (maior importância e rotatividade, representatividade de quadro de funcionários e grau de importância do conhecimento adquirido). Foi identificado que não havia uma área comum responsável pela gestão do conhecimento distribuindo-se por recursos humanos, tecnologia da informação e informática, marketing alta administração, área técnica e até mesmo por departamentos específicos nomeados como departamentos do conhecimento, cujo profissional responsável pela área possuía um cargo com nome de Chif Knowkeege Officr (CKO). Para três dos oito gestores, o assunto foi considerado multidisciplinar, envolvendo um conjunto de caracteństicas anteriormente citadas. O s oito entrevistados destacaram a relação da responsabilidade dessas atribuições com a estratégia global da firma, e não puramente da unidade de negócios. 
No referencial teórico do presente artigo é feita a menção do conhecimento como ativo estratégico, tornando a firma com maior facilidade para manter a liderança e mais difícil para os concorrentes superarem a diferença (QUINN, ANDERSO N; FINKELSTEIN; 2001). As entrevistas confirmam essa relação, estabelecendo como departamento alvo aquelas consideradas como negócio principal da empresa.

\subsection{F atores que contribuem na aplicação da gestão do co- nhecimento}

Quanto ao quinto objetivo específico, foi identificado na literatura e nas pesquisas que a aplicação da gestão do conhecimento requer alguns cuidados, de modo a garantir o ciclo do conhecimento, que Terra (2000) caracteriza como: identificação, armazenamento, disseminação, compartilhamento e uso do conhecimento organizacional. Nesse processo, algumas práticas podem auxiliar na execução de cada uma das etapas. No presente estudo, os fatores foram separados em sete itens: i) gestão de processos; ii) gestão de pessoas; iii) benhmarking iv) tecnologia dainformação; v) inovação e melhoria contínua; vi) comunicação interna estruturada, e vii) pesquisa com o cliente. Todos os entrevistados declararam que, antes de qualquer fator poder contribuir para a aplicação de um sistema de gestão baseado em conhecimento, é importante que a alta administração incorpore o processo. Nonaka (2001) destaca que na maioria das empresas o teste definitivo para a mensuração do valor dos novos conhecimentos é econômico, como o aumento de eficiência, a redução de custos, a melhora de retornos sobre o investimento. Mas, na empresa criadora do conhecimento, outros fatores mais qualitativos são igualmente importantes, como a ideia ser incorporada por toda a empresa, expressando as aspirações e as metas estratégicas da alta administração. Essa categoria levantada pela análise de conteúdo teve 139 menções distribuídas conforme se expressana Tabela 1.

Tabela 1: Fatores que contribuem na aplicação da gestão do conhecimento.

\begin{tabular}{|c|c|c|c|c|c|c|c|c|c|c|}
\hline Fator & $\mathbf{A}$ & B & C & D & $\mathbf{E}$ & $F$ & $\mathbf{G}$ & $\mathbf{H}$ & Total & $\%$ \\
\hline $\begin{array}{l}\text { Sestão de } \\
\text { rrocessos }\end{array}$ & 1 & 1 & & 2 & 1 & 2 & 1 & 11 & 19 & 14 \\
\hline $\begin{array}{l}\text { ecnologia da } \\
\text { formação }\end{array}$ & 10 & 2 & 8 & 1 & 1 & 6 & 5 & 15 & 48 & 35 \\
\hline $\begin{array}{l}\text { estão de } \\
\text { essoas }\end{array}$ & 2 & 1 & 1 & 8 & 4 & 1 & 5 & 4 & 26 & 19 \\
\hline Bendmarking & & 1 & 2 & 2 & 1 & & 1 & 4 & 17 & 9 \\
\hline $\begin{array}{l}\text { Comunicação } \\
\text { interna }\end{array}$ & & 1 & & 2 & 1 & 1 & 1 & 2 & 8 & 5 \\
\hline $\begin{array}{l}\text { Pesquisa com } \\
\text { o cliente }\end{array}$ & & 1 & & 2 & 1 & & 2 & & 6 & 4 \\
\hline $\begin{array}{l}\text { novação e } \\
\text { nelhoria } \\
\text { ontínua }\end{array}$ & 1 & 2 & & 4 & & 3 & 3 & 7 & 20 & 14 \\
\hline Total & 13 & 9 & 11 & 21 & 9 & $\mathbf{4}$ & 18 & 43 & 139 & 100 \\
\hline
\end{tabular}

Fonte: Elaborada pelos autores
Como primeiro fator que possibilita a aquisição de novos conhecimentos, gerenciando conhecimentos tácitos e explícitos, destaca-se a gestão de processos (NO NAKA, 2001; SCHNEIDERMAN, 2003; ARAÚJO; BORGES, 1999). D os oito gestores entrevistados, apenas um não apresentou ênfase nessa aplicação. D e acordo com os entrevistados, novos conhecimentos eram adquiridos na revisão constante dos processos, tornando-a um importante elo para a melhoria contínua de processos.

0 fator "pessoas" tem grande destaque no referencial teórico. Terra (2000) comenta que o sucesso de projetos e práticas de gestão do conhecimento depende não apenas de processos, mas também de pessoas. D entro desse enfoque, foram abordados na relação dos itens de gestão de pessoas e gestão do conhecimento, apontados tanto na literatura como nos levantamentos das entrevistas. A Tabela 2 ilustra a relação entre gestão de pessoas e gestão do conhecimento, assim como o resultado obtido nas entrevistas pela análise de conteúdo.

Tabela 2: Relação de itens de gestão de pessoas com gestão do conhecimento.

\begin{tabular}{l|c|c|c|c|c|c|c|c|c}
\hline \multicolumn{1}{c|}{ Fator } & $\mathbf{A}$ & $\mathbf{B}$ & $\mathbf{C}$ & $\mathbf{D}$ & $\mathbf{E}$ & $\mathbf{F}$ & $\mathbf{G}$ & $\mathbf{H}$ & $\mathbf{T}$ otal \\
\hline Líder & 0 & 1 & 3 & 4 & 1 & 1 & 2 & 5 & $\mathbf{1 7}$ \\
\hline Motivação & 1 & 0 & 1 & 3 & 2 & 0 & 4 & 19 & $\mathbf{3 0}$ \\
\hline $\begin{array}{l}\text { Rodízio de } \\
\text { Pessoas }\end{array}$ & 0 & 1 & 0 & 0 & 1 & 0 & 1 & 2 & $\mathbf{5}$ \\
\hline E-leaming & 0 & 1 & 0 & 0 & 0 & 0 & 0 & 1 & $\mathbf{2}$ \\
\hline $\begin{array}{l}\text { Universidade } \\
\text { Corporativa }\end{array}$ & 0 & 0 & 0 & 1 & 2 & 0 & 0 & 1 & $\mathbf{4}$ \\
\hline $\begin{array}{l}\text { Plano de } \\
\text { Carreira }\end{array}$ & 0 & 0 & 0 & 1 & 1 & 0 & 1 & 1 & $\mathbf{4}$ \\
\hline Líder & $\mathbf{1}$ & $\mathbf{3}$ & $\mathbf{4}$ & $\mathbf{9}$ & $\mathbf{7}$ & $\mathbf{1}$ & $\mathbf{8}$ & $\mathbf{2 9}$ & $\mathbf{6 2}$ \\
\hline \multicolumn{1}{c|}{ Total } & 0 & 1 & 3 & 4 & 1 & 1 & 2 & 5 & $\mathbf{1 7}$ \\
\hline
\end{tabular}

Fonte: Elaborada pelos autores

É importante destacar que em seis das oito empresas havia algum rodízio de pessoas entre as áreas, com o objetivo de adquirir novos conhecimentos e disseminá-los com a interação no trabalho entre as pessoas. Um dos entrevistados citou 0 programa trainœcomo uma forma de auxiliar o rodízio do funcionário em diversas áreas, possibilitando a aquisição de novos e diferentes conhecimentos e a disseminação para os antigos colaboradores dos conhecimentos criados fora da empresa.

O utro fator, a tecnologia da informação, foi o mais citado na pesquisa. Em sete das empresas entrevistadas foram adotados sistemas informatizados que permitiam a seus funcionários interação uns com os outros, criando uma cultura de compartilhamento do conhecimento eincentivando os funcionários a registrarem suas atividades, disseminando dessa forma seus conhecimentos. A penas uma das empresas não se utilizava dessa ferramenta. D estacou que o conhecimento é adquirido, 
disseminado e utilizado de outras maneiras. Em uma das multinacionais existia uma rede global de compartilhamento de conhecimento e armazenamento em comunidades de práticas, mantida por um grupo mundial de gestão do conhecimento. Nessa rede, eram mapeados os projetos feitos, a tecnologia, 0 perfil de empresas, as histórias de sucesso e os documentos (produtos, serviços, mercado e competidores). Uma vez identificada uma necessidade com relação a algum conhecimento específico, podia-se pesquisar nesse sistema se havia algum colaborador ao redor do mundo que possuísse esse conhecimento. Nesse momento, trocas de informação poderiam surgir, disseminações e compartilhamento de conhecimento, contribuindo para o projeto em questão.

Segundo Terra(2000), o uso das tecnologias de informática é necessário, mas está longe de ser suficiente. 0 autor destaca que a gestão do conhecimento focada prioritariamente nos investimentos em infraestrutura tende a resultar em fracasso, pois conhecimento é informação interpretada, o que faz com que a simples transferência de informação não aumente 0 conhecimento ou a competência. Nesse enfoque, uma das empresas também se utiliza de um sistema de tecnologia. É permitido estudar todo 0 histórico de projetos, o que foi estudado, quais os pontos abordados, qual o aprendizado tirado com aquela experiência, os vídeos, as fotografias e os depoimentos de pessoas que participaram do projeto. D essa forma, o profissional entrevistado destacou que o uso da tecnologia da informação propiciaidentificar, na empresa, pessoas que conheçam determinado assunto. Ela era uma incentivadora para a disseminação efetiva do conhecimento. Para esse entrevistado, era na próxima etapa, no encontro entre as pessoas, que $70 \%$ do conhecimento iria ser trocado.

A comunicação interna estruturada estimula o trabalho em equipe e departamentos da empresa a trocarem conhecimentos adquiridos. Todos os entrevistados declararam que suas empresas possuíam um canal de disseminação de informação e conhecimento, sendo que o mais utilizado é o jornal institucional, que procurou abordar os últimos acontecimentos e os objetivos estratégicos da firma.

A técnica de benhmarking de acordo com Garvin (2001), é uma contínua busca por melhores práticas interna e extemamente a organizações com o sentido de acelerar a aprendizagem organizacional. Segundo esse autor, a empresa que pratica essa técnica está buscando adquirir conhecimento no mercado e poderá agregá-lo no dia a dia da empresa. Nas entrevistas, há uma constante preocupação por essa prática, aplicada tanto externa quanto internamente. Em quatro das oito empresas, a técnica era utilizada nas equipes de venda, quando um vendedor que ganhou destaque e conseguiu algum retorno melhor dividia a boa prática com os demais. Em algumas delas haviaum envolvimento maior por parte de prêmios e divulgações que possibilitasse maior destaque a esse colaborador. Em duas multinacionais que participaram da entrevista, havia uma constante busca de benchmarkingem suas unidades internacionais.

Outra maneira de adquirir novos conhecimentos, destacados na entrevista, era por meio da pesquisas com os clientes. E ssa estratégia para conhecimento do mercado, destacada nas entrevistas, é também abordada por Garvin (2001) e Hayes (2001). D e acordo com os autores, é possível adquirir novos conhecimentos que poderão ser compartilhados com a equipe, auxiliando nas tomadas de decisões. Apesar de destacadas como importantes, apenas três das oito empresas reconhecem utilizar-se dessas práticas. Para essas três empresas, as conclusões tiradas com as análises eram divulgadas para os colaboradores, com o objetivo principal de fortalecer o relacionamento entre as empresas e os parceiros de negócios. Tanto o primeiro como 0 segundo autor citado no início deste parágrafo avaliam que esse conhecimento adquirido é essencial para a formação de opinião sobre segmentos de mercado e linhas de serviços específicas. Nesse sentido, nas entrevistas, os gestores destacaram 0 aprendizado mútuo, proveniente da pesquisa.

A inovação e a melhoria contínua foram outros dois pontos abordados. Leonard e Straus (2001) destacam que o desafio gerencial é usar os insightsprovenientes das inovações para criar novos processos e encorajar novos comportamentos que contribuirão para criar novos conhecimentos. Três dos oito gestores entrevistados destacaram a preocupação da inovação contínua a fim de trazer conhecimento no desenvolvimento de seus produtos e serviços. Com relação à melhoria contínua, $\mathrm{G}$ arvin (2001) afirma que os programas contínuos normalmente envolvem uma série ininterrupta de pequenos experimentos, destinados a produzir ganhos incrementais no conhecimento. Ele afirma que são muito comuns nas fábricas e oficinas. A entrevista mostra essa aplicação, principalmente nas empresas de engenharia e tecnologia. O s entrevistados abordam o sistema de qualidade como um importante elo entre a qualidade e os novos conhecimentos adquiridos.

\section{$4.40 \mathrm{~s}$ benefícios competitivos das práticas de gestão do conhecimento}

0 último objetivo específico se referia aos resultados obtidos, às ferramentas utilizadas para mensurar os benefícios atribuídos às práticas de gestão do conhecimento eà identificação desse recurso como estratégico. 0 objetivo principal eraidentificar se o conhecimento é reconhecido pelas empresas como uma fonte de vantagem competitiva. É nele que se fecha o ciclo de avaliação deste estudo e permite a conexão com o objetivo principal. Como os cinco objetivos específicos anteriores associam-se com as práticas de gestão do conhecimento, primeiro construto desta pesquisa, este último refere-se ao segundo construto, a capacidade de competição.

A análise das entrevistas sugeriu que os gestores tinham dificuldadeao estabelecer algumasmedidas de mensuração. Apesar de citarem a subjetividade com relação à mensuração dos resultados, quatro deles informaram que as suas empresas se utilizavam de ferramentas formais parajustificar as iniciativas. Um dos entrevistados afirmou usar o Balancel Sccrearde contabilizava a quantidade de acessos por ferramentas de tecnologia de informações. $\mathrm{O}$ utra firma apenas utilizava a quantidade de acessos, uma controlava o nível de motivação, e uma quarta empresa quantificava o número de ideias utilizadas efetivamente daquelassugeridas em um banco de dados de sugestões. 
Todos os gestores entrevistados identificaram o conhecimento com um recurso estratégico e afirmaram que ele erafonte de vantagem competitiva na firma em que atuava. Um dos gestores entrevistados enumerou os benefícios criados por essas práticas e afirmou que a empresa consegue se diferenciar no mercado e cortar custos significativos de produção. O utro entrevistado afirmou que o conhecimento, no futuro, tendia a ser uma condição essencial para a sobrevivência das empresas no mercado. 0 Q uadro 6 ilustra os resultados da análise de conteúdo quanto aos resultados advindos do processo de gestão do conhecimento para as empresas pesquisadas.

\begin{tabular}{|l|c|c|c|c|c|c|c|c|c|}
\hline Resultados & A & B & C & D & E & F & G & H & Total \\
\hline $\begin{array}{l}\text { Diminuição de } \\
\text { custos }\end{array}$ & Sim & & Sim & & & & Sim & $\mathbf{3}$ \\
\hline $\begin{array}{l}\text { Maior } \\
\text { agilidade }\end{array}$ & & Sim & & & & & Sim & Sim & $\mathbf{3}$ \\
\hline $\begin{array}{l}\text { Mais preparo } \\
\text { da equipe } \\
\text { comercial }\end{array}$ & & Sim & & & & & & $\mathbf{1}$ \\
\hline Mais inovação & & & Sim & & & & & $\mathbf{1}$ \\
\hline $\begin{array}{l}\text { Ganho de } \\
\text { mercado }\end{array}$ & & & $\operatorname{Sim}$ & $\operatorname{Sim}$ & & $\operatorname{Sim}$ & & $\operatorname{Sim}$ & $\mathbf{4}$ \\
\hline $\begin{array}{l}\text { Aumento de } \\
\text { qualidade }\end{array}$ & & & & $\operatorname{Sim}$ & & & & $\operatorname{Sim}$ & $\mathbf{2}$ \\
\hline $\begin{array}{l}\text { Aumento de } \\
\text { motivação }\end{array}$ & & & $\operatorname{Sim}$ & $\operatorname{Sim}$ & & $\operatorname{Sim}$ & $\operatorname{Sim}$ & $\mathbf{4}$ \\
\hline $\begin{array}{l}\text { Melhora na } \\
\text { satisfação do } \\
\text { cliente }\end{array}$ & & & $\operatorname{Sim}$ & & & & $\operatorname{Sim}$ & $\mathbf{2}$ \\
\hline
\end{tabular}

Quadro 6: Resultados obtidos com a gestão do conhecimento Fonte: Elaborado pelos autores, com base nos dados da pesquisa.

Esses itens abordados pelos gestores podem ser interpretados como produtos e serviços oferecidos aos clientes a partir de fatores tidos e controlados pela empresa, ou seja, recursos (AMIT; SCHOEMAKER, 1993). Os gestores afirmaram espontaneamente que a gestão do conhecimento possibilita alcançar esses benefícios. Essa melhoria no desempenho visualizada pelos itens abordados está relacionada ao recurso e à competência organizacional, ao conhecimento, oferecendo uma estrutura própriapara o estudo davantagem competitiva (BARNEY, 2002; DIERICKX e CO OL, 1989; PETERAF, 1993).

Esses itens podem ainda ser vistos so $\mathrm{b}$ a perspectiva de Barney (2001). 0 autor aborda o recurso como fonte de vantagem competitiva ao ser valioso para o cliente, com capacidade para a firma explorar, de difícil imitação, e raro. A diminuição de custos, a melhora na eficiência, a inovação e a melhoria nos produtos, e 0 aperfeiçoamento no serviço ao cliente se constituem ainda de fatores que necessitam de um longo caminho de aprendizado (pathdependene) (BARNEY, 2001).

\section{LIMITAÇÕES, CONCLUSÕES E DIRECIONA- MENTOS FUTUROS}

O estudo de gestão do conhecimento aqui apresentado foi limitado aos setores de serviços e da indústria. As empresas foram selecionadas por conveniência devido à facilidade de acesso dos pesquisadores e, portanto, não reuniu empresas da mesma indústria. Variáveis como o porte de cada empresa, medido pelo número de funcionários ou faturamento, não foram fatores de seleção, podendo também constituir uma limitação do estudo. A seleção final das empresas na pesquisa foi feita pela afirmação de que havia a aplicação de um sistema de gestão do conhecimento, independentemente da fase ou do entendimento por parte dos gestores do que era esse construto, podendo abranger outra limitação.

Considerando-se o objetivo do presente estudo, que era identificar a percepção dos gestores sobre a relação entre as prá ticas de gestão do conhecimento e a de uma fonte de vantagem competitiva, pode-se atribuir que ele foi alcançado. O s gestores entrevistados reconhecem que há uma relação entre as duas variáveis, sendo identificados também diversos fatores que contribuem e são utilizados pelas empresas no ciclo da gestão do conhecimento: geração, identificação, armazenamento, disseminação, compartilhamento e uso do conhecimento organizacional. As medidas de desempenho citadas não podem ser configuradas como medidas reconhecidas. Contudo, trazem indícios com os benefícios obtidos a partir da gestão do conhecimento como, por exemplo, a redução de custos, a agilidade de processos e 0 aumento de satisfação dos clientes.

Esta pesquisa identificou que os entrevistados têm, em geral, receio ao afirmar que a firma em que trabalham não há processos e métricas que possam afirmar e garantir a aplicação da gestão do conhecimento. Poucos gestores apresentavam uma visão sólida dos conceitos abordados, tendo em geral, algumas ideias particulares interpretadas por eles mesmos. Essa subjetividade pôde ser percebida principalmente na identificação dos processos aplicados em cada firma. Em algumas empresas a preocupação central e o entendimento de gestão do conhecimento se limitam à disseminação e ao compartilhamento do conhecimento; em outras, apenas na identificação. Poucas reconhecem o ciclo completo e realizam processos para garantir que 0 ciclo todo esteja sendo seguido.

No cruzamento entre o referencial teórico e as entrevistas foram identificados sete fatores que contribuem para que as etapas da gestão do conhecimento sejam satisfeitas: gestão de processos; gestão de pessoas (subdividido em liderança, plano de carreira e rodízio de pessoas, incentivos à motivação, aprendizagem organizacional e universidade corporativa); bentmarking tecnologia da informação; inovação e melhoria contínua; comunicação interna estruturada, e pesquisa com o cliente. Pode-se concluir que cada um desses fatores contribui em determinada etapa do processo de gestão do conhecimento.

A gestão do conhecimento nas organizações passa pela compreensão das características, recursos e competências internas e intrínsecas de cada firma e, também, pelo entendimento 
das necessidades individuais e coletivas associadas aos processos de criação e aprendizado. As entrevistas sugerem que o desafio de produzir mais e melhor vai sendo suplantado pelo desafio, permanente, de criar novos produtos, serviços, processos e sistemas gerenciais. Por sua vez, a velocidade das transformações e a complexidade crescente dos desafios não permitem mais concentrar esses esforços em alguns poucos indivíduos ou algumas áreas das organizações.

O presente estudo se torna um importante enfoque na análise da RBV sob o aspecto da VRIO, sugerindo que a posição das empresas está baseada em seus recursos e suas capacidades singulares valiosas, raras e difíceis de imitar. As entrevistas evidenciaram uma associação entre as atividades de gestão do conhecimento e a obtenção de vantagem competitiva.

Como sugestão para estudos futuros sugere-se uma pesquisa de caráter quantitativo envolvendo uma ou duas atividades econômicas de indústria, comércio ou serviços. Nesse caso, o objetivo seria a aplicação de um questionário estruturado e fechado, desenvolvido a partir dos achados deste estudo qualitativo e que permitiria avaliar o relacionamento entre os dois construtos de pesquisa, segundo modelos estatísticos relacionados com análises uni, bi ou multivariada.

\section{RE FE RÊ N CIAS}

ACED O, Francisco José; BARRO SO, Carmen; GALAN, José Luis. The resource-based theory. Dissemination and main trends. Strategic Managament Jaurnal. n. 27, p. 621-636, 2006.

ALAVI, M.; LEIDNER, D. E. Review: knowledge management and knowledge management systems: conceptual foundations and research issues. MS Quartely. v. 25, n. 1, p. 107-133, 2001.

AMIT, R.; SCHOEMAKER, P. J. H. Strategic assets and organizational rent. Strategic Management Jaumal. v.14, n. 1, p.33-46, Jan 1993.

ARAUJO, R. M.; BO RG ES, M. R. S. Sobrea aplicabilidade de sistemas de workflow no suparte a processos de sattware, San Jose, Costa Rica, p.417-428, Mar 1999.

ANSOFF, H. I. Corparate strategy. Harmondsworth: Penguin, 1965. BARD IN, L. Análise de contára Lisboa: Edições 70, 1997.

BARNEY, J. Gainingand sustaining compditiveadkantage 2nd ed. N ew Jersey: Prentice Hall, 2002.

. Resource-based theories of competitive advantage: a ten year retrospective on the resource-based view. Journal of Management, v. 27, n.6, p.643-650, 2001.

BECKMAN, T. A methodology for knowledge management. In LIEBOWITZ, Jay. Knowkedgemanagement handbodk. CRC Press, 1999.

BRITO, L. A. L.; VASCONCELOS, F. Vantagem competitiva: 0 construto e a métrica. Revista de Administração de Empresas, 2004.

BROUTHERS, Keith D.; BROUTHERS, Lance E.; WERNER, Steve. Resource-based advantages in international context. Joumal of Management, v. 34, n. 2, p. 189-217, April, 2008.

CHIA, Robert; HOLT, Robin. On managerial knowledge. Management L@ming V.39, n.2, p. 141-158, 2008.

D IERICKX, I.; COOL, K. Asset stock accumulation and sustainability of competitive advantage. Management Saience v.35, n.12, p.1504 - 1511, 1989.

EISENHARD T, K. M.; SANTOS, F. M. Knowledge-based view: anew theory of strategy? In: EARL, Michael. Knowledge management strategies: towards a taxonomy. Jaumal of Management Infomation Systems 18(1), Summer, 2001.

GARVIN, D. A. Construção da organização que aprende. In: SERRA, A. C. Gestão do conhecimento: on knowledge management. Harvard Business Review 5ed. Rio de Janeiro: Campus, 2001.

G HEMAWAT, P. Sustainable advantage. Harvard Business Review, v.64, n.5, p.53-58, 1986.

GO URLAY, Stephen. Tacit knowledge: the variety of meanings in empirical research. 5th OKLC Conference Innsbrudk, April, 2004.

HAYES, B. E. Meeindo a satisfação do diente 1 ed. Rio de Janeiro: Q ualitymark, 2001.

HAWLEY, A. Human Ecology. In: D avid Sills (ed.). International engydopedia of the soial science p. 328-37. New York: MacMillan, 1968.

LEONARD, D.; STRAUS S. Aproveitando todo o cérebro da empresa. In: SERRA, A. C. G estão do conhecimento: on knowledge management. Harvard Business Review 5ed. Rio de Janeiro: Campus, 2001.

NO NAKA, I. A empresa criadora do conhecimento. In: SERRA, A. C. G estão do conhecimento: on knowledge management. Harvard Business Review Rio de Janeiro: Campus, 2001.

; TAKEUCHI, H. Criação de conheeimento na empresa: com as empresas japanesas gerama dinâmica da inovaçãa 3ed. Rio de Janeiro: Campus, 1997.

PETERAF, M. The cornerstones of competitive advantage - a resource-based view. Strategic Management Journal, v. 12, n.3, p.95117, 1993.

POWE LL, W.; D IMAG G IO, P. J. The new institutionalism in arganizational analysis Chicago: The University of Chicago Press, 1991. PORTER, M. E. Competitive strategy: techniques for analysing industries and competitors. New York: Free Press,1980.

. Compeitive advantage creating and sustaining superior performance. New York: Free Press, 1985.

DAVENPORT, T. H., PRUSAK, L. Working knowledge how organizations manage what they know. 1 ed. Boston, Harvard Business School Press, 1998.

QUINN, B. Intelligent enterprise a knowledge and service based paradigm for industry. New York: The Free Press, 1992.

; AND ERSON P.; FINKELSTEIN, S. G erenciando 0 intelecto profissional: extraindo o máximo dos melhores. In: SERRA, A. C. G estão do conhecimento: on knowledge management. Harvard Business Review 5ed. Rio de Janeiro: Campus, 2001.

RICHARD SO N, R. J. et al. Pesquisa sodal: métodos e técnicas. 3 ed. São Paulo: Atlas, 1999.

RUMELT, R. How does industry matter? Strategicmanagenentjaumal, v. 12, n.3, p.167-169, 1991.

SCHERER, F. M; ROSS, D. Industrial market structure e economic pefommane, 2003. Boston: Houghton Mifflin Company, 1991.

SCHNEID ERMAN, A. M. Process management 2000, 2003. D isponível em <http:/ / www.schneiderman.com>. Acesso em 15 mar. 2003.

SPENCER, J. C.; GRANT, R. M. Knowledge and the firm: overview. Strategic Management Jaumal, Winter Special Issue, v.17,n.1, p.5-9, 1996.

TERRA, J. C. C. Gestão do conhecimenta o grande desafio empresarial. Rio de Janeiro: Negócios, 2000.

TSO UKAS, Haridimos. D o we really understand tacit knowledge? Knoutedge Econamy and Socidy Seminar, 2002 
YANG, Monica; HYLAND, Mary Anne. Who do firms imitate? A multilevel approach to examining sources of imitation in the choice of mergers and acquisitions. Joumal of Managment, v.32 n. 3, June, p. 381-399, 2006.

WERNERFELT, Birger. A resource-based view of the firm. Strategic Managenent Jaumal, Vol. 5, Issue 2, A pr. - Jun., p. 171-180, 1984

WIIG, K. M. Knowkedgemanagement: an emerging discipline rooted in a long history, 2003. D isponível em <http:/ / www.krii.com>. Acesso em 15 mar. 2003.

WILLIAMSO N, O. E. Strategizing, economizing, and economic organization. StrategicManagement Jaumal, Winter Special Issue, v.12, n.1, p. 75-94, 1991.

Data de Submissão: 02/ 01/ 2007

Data de Aprovação: 18/ 10/ 2009 